\title{
Asymmetric optical radiation pressure effects on liquid interfaces under intense illumination
}

\author{
Alexis Casner, $1,2, *$ Jean-Pierre Delville, ${ }^{1,+}$ and Iver Brevik ${ }^{3,+}$ \\ ${ }^{1}$ Centre de Physique Moléculaire Optique et Hertzienne, \\ UMR CNRS/Université 5798, Université Bordeaux I, \\ 351 Cours de la Libération, F-33405 Talence cedex, France \\ ${ }^{2}$ Present address: Département Conception et Réalisation des Expérimentations, \\ CEA-DAM Ile de France, BP 12, F-91680 Bruyères-le-Châtel, FRANCE \\ ${ }^{3}$ Department of Energy and Process Engineering, \\ Norwegian University of Science and Technology, N-7491 Trondheim, Norway.
}

(Dated: June 25, 2021) 


\begin{abstract}
Deformations of horizontal liquid interfaces by optical radiation pressure are generally expected to display similar behaviors whatever the direction of propagation of the exciting laser beam is. In the present experiment we find this expectation to be borne out, as long as the cw laser illumination is moderate in strength. However, as a striking contrast in the case of high field strengths, we find that either a large stable tether can be formed, or else that a break-up of the interface can occur, depending on whether the laser beam is upward or downward directed. Physically, the reason for this asymmetry can be traced to whether total reflection can occur or not. We also present two simple theoretical models, one based on geometrical optics, the other on wave optics, that are able to illustrate the essence of the effect. In the case leading to interface disruption our experimental results are compared with those obtained by Zhang and Chang for water droplets under intense laser pulses [Opt. Lett. 13, 916 (1988)]. A key point in our experimental investigations is to work with a near-critical liquid/liquid interface. The surface tension becomes therefore significantly reduced, which thus enhances the magnitude of the stationary deformations induced.
\end{abstract}

PACS numbers:

*Electronic address: alexis.casner@cea.fr

${ }^{\dagger}$ Electronic address: jp.delville@cpmoh.u-bordeaux.fr

${ }^{\ddagger}$ Electronic address: iver.h.brevik@mtf.ntnu.no 


\section{INTRODUCTION}

Since the seminal work of Ashkin and Dziedzic [1] on the deformation of transparent liquid free-surfaces induced by laser waves, the optical radiation pressure has been recognized as very appealing to locally manipulate liquid interfaces. The first developments exploited essentially the interface bending for optical applications in adaptative lensing [2], formation of surface relief holographic gratings [3], or control of morphology-dependent resonances 4]. Recently, radiation pressure effects have received renewed interest in connection with nano/bio-technologies, as a non-intrusive tool to probe microscopic surface properties of soft materials including cell stretching [5, 6] or membrane and interface viscoelasticity [7, 8, 9, 10]. However, the bending of a fluid interface by the optical radiation pressure is generally weak, and all these experiments were thus essentially limited to the investigation of the linear regime in deformation.

The "linear regime" concept here ought to be defined more precisely, as there are actually two kinds of nonlinearities to be recognized. First, hydrodynamic nonlinearity occurs if the slope of the liquid surface bulge is large. In this context it is instructive to consider the Zhang-Chang droplet experiment [11]. These authors presented impressive pictures of the surface shape distortions in the front and in the rear region of a micrometer-sized water drop. When illuminated with a strong laser pulse, droplet disruption was observed at the rear. If the equilibrium radius of the droplet is $a$ and the surface elevation is $h$, linear hydrodynamic wave theory is known to apply if the transverse gradient of $h$ is much less than one. In the case of a $100 \mathrm{~mJ}$ pulse, the calculation of the Zhang-Chang experiment (Fig. 2 in Ref. 12) gave under typical conditions $h / a=0.02$ at the front and $h / a=0.3$ at the rear. Accordingly, hydrodynamic linear theory should be expected to apply at the front 
but is obviously not valid at the rear.

The other kind of nonlinearity that may occur is of electromagnetic nature, caused by a large value of the field strength. Generally, the ratio between the third-order and the firstorder susceptibilities is written as $\chi^{(3)} / \chi^{(1)}=E_{0}^{-2}$, where $E_{0}$ is for most materials of order of $10^{11} \mathrm{~V} / \mathrm{m}$ (see Ref. 13). Consider again the Zhang-Chang experiment. In case of the $100 \mathrm{~mJ}$ pulse the incident intensity at the centerline of the beam was $0.29 \mathrm{GW} / \mathrm{cm}^{2}$, which corresponds to an electric field of about $10^{7} \mathrm{~V} / \mathrm{m}$. As the droplet acts as a lens, the field in the rear region is higher, perhaps by a factor of 2 or more. However, on the whole we find it unlikely that the field strength was strong enough to affect the susceptibility significantly in the Zhang-Chang experiment. We will consider similar conditions here. To be precise: we will assume hydrodynamic nonlinearity, but electrodynamic linearity.

The background for the present work is the following. Whereas the linear regime is well understood and also delineated experimentally [14], the nonlinear behavior at strong laser illumination is still at an early stage. Some success has so far been achieved in the theoretical description of the weakly nonlinear regime of deformation [12, 15]. However such a scheme, based as it is on linear wave theory, is clearly insufficient to explain the very large asymmetry observed. This brings us to the primary aim of the present research, namely to show the existence of these large deformations experimentally, to explore the physical reason for the asymmetry, and finally to present a simple theoretical model whereby the essence of the phenomenon can be described. To the best of our knowledge, this effect is observed for the first time at steady state under cw laser excitation, i.e. in a situation particularly favorable for quantitative investigations. Even if the asymmetry as such is not novel, we think that the very large magnitude of it, is.

We choose to work experimentally with liquid surfaces that are initially flat. Considering 
a laser beam coming either from above or from below on the meniscus we are thus able to analyze the surface deformations without entering into the complications arising from the lens effect in the case of curved surface - cf. the striking demonstration of the last-mentioned effect in the Zhang-Chang experiment.

We point out that the distortion of the liquid surface depends in general on the combined effect of gravity and surface tension. The equation of motion for the height $h(r)$, where $r$ is the radius in cylindrical coordinates, is given at steady state by:

$$
\left(\rho_{1}-\rho_{2}\right) g h(r)-\sigma \frac{1}{r} \frac{d}{d r}\left(\frac{r h^{\prime}(r)}{\sqrt{1+h^{\prime}(r)^{2}}}\right)=\Pi(r)
$$

where $\rho_{1}, \rho_{2}$ are the densities of the two liquids, $\sigma$ the surface tension and $\Pi(r)$ the optical radiation pressure. In the linear regime of deformation, $h^{\prime}(r) \ll 1$ and the radiation pressure value is taken at normal incidence (see for instance Eq. (1) in Ref. 14). Then for an exciting beam in the $T E M_{00}$ mode, the solution for $h(r)$ can be calculated using a Fourier-Bessel transform and contains the capillary gravity wave frequency $\Omega(k)=\sqrt{\frac{\rho_{1}-\rho_{2}}{\rho_{1}+\rho_{2}} g k+\sigma \frac{k^{3}}{\rho_{1}+\rho_{2}}}$ as a central parameter (cf. also p. 171 in Ref. 16). However by comparing the relative effect of gravity (buoyancy) to surface tension, one defines an optical Bond number Bo as $B o=\left(\omega_{0} / l_{C}\right)^{2}$, where $\omega_{0}$ is the beam waist and $l_{C}=\sqrt{\sigma /\left(\rho_{1}-\rho_{2}\right) g}$ is the capillary length of the interface. When $B o \ll 1$, and it will be the case in the following experiments, then gravity turns out to be negligible for the surface distortion. The centerline height is in this case inversely proportional to the surface tension $\sigma$, and is thus considerably enhanced when $\sigma$ is a small quantity. A closer discussion on this point is 
given in Ref. 14.

\section{EXPERIMENTS}

\section{A. Set-up}

Experiments were performed in a water-in-oil microemulsion (stable suspension of surfactant-coated water nano-droplets, called micelles, dispersed in an oil-rich continuum). Its composition and some of its characteristics have already been described previously [14]. For a temperature $T>T_{C}$, where $T_{C} \simeq 308 \mathrm{~K}$ is a critical temperature, the mixture separates in two micellar phases $\Phi_{1}$ and $\Phi_{2}$ of different concentrations. Since the density (resp. index of refraction) of water is larger (resp. smaller) than that of oil, the micellar phase $\Phi_{1}$ of larger concentration is located below the low micellar concentration phase $\Phi_{2}$ while its refractive index $n_{1}$ is smaller than $n_{2}$ of $\Phi_{2}$. The main advantages of our medium are the extremely weak surface tension of the liquid meniscus separating the two phases (typically $10^{6}$ times smaller at $T-T_{C}=3 \mathrm{~K}$ than that of the water/air free surface) and its very low residual absorption at the wavelength used. As a consequence interface deformations can easily be monitored by continuous laser waves without disturbing thermal couplings or non-linear bulk effects [17]. Moreover the vicinity of a critical point ensures the universality of the observed phenomena, because our mixture belongs to the universality class $(\mathrm{d}=3, \mathrm{n}=1)$ of the Ising model [14]. It allows also us to evaluate the experimental parameters of our system according to the following scaling laws for the surface tension $\sigma$ and the density contrast of the two phases

$: \sigma=\sigma_{0}\left(\frac{T-T_{C}}{T_{C}}\right)^{2 \nu}$, with $2 \nu=1.26$ and $\sigma_{0}=10^{-4} \mathrm{~J}^{2} \mathrm{~m}^{-2} ; \Delta \rho=\rho_{1}-\rho_{2}=\Delta \rho_{0}\left(\frac{T-T_{C}}{T_{C}}\right)^{\beta}$, with $\beta=0.325$ and $\Delta \rho_{0}=285 \mathrm{~kg} \cdot \mathrm{m}^{-3}$. For the refractive index contrast $\Delta n=n_{1}-n_{2}$, as the two phases $\Phi_{1}$ and $\Phi_{2}$ are of very close composition, we assume the Clausius-Mossotti 
relation to be valid [14]: $\Delta n \simeq\left(\frac{\partial n}{\partial \rho}\right)_{T} \Delta \rho$ with $\left(\frac{\partial n}{\partial \rho}\right)_{T}=-1.2210^{-4} \mathrm{~m}^{3} \cdot \mathrm{kg}^{-1}$. The Table 1 gives the values of these parameters, together with the values of the absorption and thermal coefficients of our medium.

The experimental set-up is shown in Fig.1. The mixture is enclosed in a thermoregulated spectroscopic cell and the temperature is chosen above $T_{C}$ to reach the two-phase equilibrium state. The bending of the liquid-liquid meniscus is driven by a linearly polarized $T E M_{00} \mathrm{cw}$ $A r^{+}$laser (wavelength in vacuum, $\lambda_{0}=514 \mathrm{~nm}$ ) propagating either upward or downward along the vertical axis. The beam is focused on the interface by an objective lens (Leitz 10X, N.A. 0.25). In the following $P$ is the beam power and the beam-waist $\omega_{0}$, evaluated at $\frac{1}{e^{2}}$, is adjusted by changing the distance between a first lens $\mathrm{L}$ (focal length $\mathrm{f}=1 \mathrm{~m}$ ) and the focusing objective.

\section{B. Results}

Typical interface deformations induced for identical experimental conditions $\left(T-T_{C}=\right.$ $3 K$ and $\left.\omega_{0}=5.3 \mu m\right)$ are presented on Fig. 2 for upward and downward directed beams. Since $n_{1}<n_{2}$, the radiation pressure acts downwards toward the less refractive medium, regardless of the direction of propagation of the laser [10, 16]. Fig. 3 shows the variation of the centerline height $h_{0}=h(r=0)$ versus beam power $P$ in both cases. As expected and already observed in experiments [14], $h_{0}$ is linear in $P$ at low beam power. This regime corresponds respectively to $P \leq 225 \mathrm{~mW}$ and $P \leq 300 \mathrm{~mW}$ in the two examples presented. Then when $P$ increases, $h_{0}$ gradually deviates from linearity. The deformation switches from the classical bell shape to a stable tether shape in the upward case (see the last four pictures on Fig. 2a)). The behavior is radically different in the downward excitation case. The 
deformation suddenly looses stability and $h_{0}$ diverges above a well-defined onset power $P_{S}$ (see for instance the two last pictures at $P=P_{S}=400 \mathrm{~mW}$ on Fig. 2b)). This instability gives birth to a liquid jet that self-traps the beam and emits droplets at its end (Fig. 2c).

\section{THEORETICAL DISCUSSION}

\section{A. The Importance of Total Reflection; scaling relation for the onset power $P_{S}$}

Actually, the reasons for this symmetry breaking can be understood from a very simple physical argument. As the beam propagates from the large to the low refractive phase in the downward case, total reflection of light at the interface can be reached. When this occurs, there is a dramatic concentration of light energy towards the tip of the bulge which consequently becomes unstable. We have already demonstrated that, under a wide range of scaling conditions, the measured onset power of the instability leading to the liquid jet is in complete accordance with the onset power $P_{S}$ defined by the condition of total reflection 18]:

$$
P_{S}=\frac{1.121 \pi}{0.715 \sqrt{2}} \frac{n_{1}}{n_{2}}\left(1+\frac{n_{1}}{n_{2}}\right) \frac{\sigma c}{n_{2}-n_{1}} \omega_{0}
$$

where $\sigma$ as before is the meniscus surface tension and $\mathrm{c}$ is the light velocity in vacuum. The advantage of working with critical fluids is another time obvious here, as $P_{S}$ scales as $\frac{\sigma}{n_{2}-n_{1}} \propto\left(T-T_{C}\right)^{0.93}$ and therefore vanishes close to $T \simeq T_{C}$.

For the opposite direction of propagation, the induced tethers (up to $60 \mu m$ on Fig. 2a)) 
are surprising. A coupling still exists between the laser propagation and the deformation because the bulge acts as a soft lens [17]. However, in this case the beam is focused inside the deformation and no amplification mechanism therefore occurs for the intensity experienced by the tip of the bulge. These unusual nonlinear shapes deserve further theoretical investigations. To our knowledge, they remain so far unexplained.

\section{B. A Simple Two-dimensional Geometrical Optics Model}

We find it worthwhile to point out that it is possible to get a physical picture of the essence of the asymmetry in the downward/upward cases of the laser beam, without having to take into account the complex circular geometry of the tether. Before embarking on calculations, one may ask to what extent an approximate picture in terms of geometrical optics could be adequate. The important parameter distinguishing between wave optics and geometrical optics is, for a sphere of radius $a$,

$$
\alpha=2 \pi a / \lambda_{0}
$$

The distinction between the two cases is not sharp, but in practice it is usually safe to work in terms of geometrical optics if $\alpha \geq 80$, as discussed, for instance, in Ref. 12 . Assuming the highly curved region at the tip of the distortion to correspond roughly to a sphere of radius $a=10 \mu \mathrm{m}$ we obtain $\alpha \simeq 120$ for $\lambda_{0}=514 \mathrm{~nm}$. The geometrical optics picture should according to this estimate be quite safe. Even if the radius becomes halved, 
the resulting value $\alpha \simeq 60$ is most likely to be sufficient to justify the use of geometrical optics in our case.

Consider then the following simple model. Replace the tether with a symmetrical two-dimensional wedge, with an opening angle $2 \beta$ facing upwards. Assume that an incident vertical ray in the downward direction falls from the optical dense medium (2) towards one of the wedge surfaces, gets reflected towards the second surface, and is thereafter reflected back in the upward vertical direction (see Fig. 4). This symmetric ray pattern becomes accomplished if we choose $\beta=45^{0}$, and assign the same value to the angle of incidence $\theta_{2}$. The surface pressure $\Pi$ caused by one single reflection/refraction is given by the generic formula [19]:

$$
\Pi\left(\theta_{i}\right)=\frac{n_{i} I}{c} \cos ^{2} \theta_{i}\left\{1+R-\frac{\tan \theta_{i}}{\tan \theta_{t}} T\right\},
$$

where the index $\mathrm{i}$ refers to incidence and t to transmission, $I$ being the incident intensity. This formula can be derived either by integration of the normal component of the volume force density $\mathbf{f}=-\frac{1}{2} E^{2} \nabla \varepsilon$ across the boundary region of the dielectric, or alternatively by considering the normal component of Maxwell's stress tensor directly [16]. The coefficients of reflection and transmission, $R$ and $T$, satisfy the condition $R+T=1$. They can be found, for instance, on p. 496 in Stratton's book [20]. The expression Eq. (3) holds regardless of the state of polarization of the beam. In practice, we usually insert the expressions for $R$ and $T$ corresponding to the TM or TE polarizations.

Consider first the downward incident ray, for which $\theta_{i}=\theta_{2}$ and $n_{i}=n_{2}$. Without 
losing the essence of the problem we can make the simplifying assumption that this ray corresponds to the onset of total reflection. Then from Snell's law, $n_{2} / n_{1}=\sqrt{2}$. (We are thus choosing refractive indices different from those actually used in our experiment; this only to make the argument as simple as possible.) The two reflections from the wedge surfaces contribute with equal weight to the pressure, since in this case $R=1, T=0$. We calculate the vertical force coming from $\Pi\left(\theta_{2}\right)$ over a length $l$ of the surface $(l$ reckoned from the cusp), multiply by 2 because of the two surfaces, and define a mean vertical pressure $\bar{\Pi}_{z}(\downarrow)$ by dividing with the effective cross section $l \sqrt{2}$ for the considered part of the beam:

$$
\bar{\Pi}_{z}(\downarrow)=\frac{n_{2} I}{c}
$$

For the reverse case of an upward directed incident ray, assumed to have the same intensity $I$, there is no total reflection to be taken into account. The angle of incidence, now called $\theta_{1}$, is $45^{0}$ as before, whereas now $n_{i}=n_{1}$. Assuming for definiteness the polarization to be in the plane of incidence (TM wave), we calculate $R=0.005, T=0.995$, and find that $\Pi\left(\theta_{1}\right)=0.359 n_{1} I / c$. The corresponding mean vertical pressure becomes

$$
\bar{\Pi}_{z}(\uparrow)=\frac{0.359 n_{1} I}{c}
$$

The pressure ratio is 


$$
\frac{\bar{\Pi}_{z}(\downarrow)}{\bar{\Pi}_{z}(\uparrow)} \simeq 4
$$

showing that the present crude model is able to predict a significant higher radiation pressure when the beam is downward directed. It is therefore physically understandable that strong-field effects like the emission of droplets occur in the downward case, i.e. when incidence occurs from the optically denser medium.

\section{Axially Symmetric Wave-Theoretical Model}

Quite justifiably, one may argue that the above model gives an over-simplified description of what happens physically. The real situation is after all axially symmetric. To give a detailed wave-optical description of the free surface displacement as a function of radius in cylindrical coordinates, would be rather complicated. Even the description of the simple case of a monochromatic wave propagating inside a cylindrical medium of infinite length is

quite complicated (cf., for instance, Section 9.15 in Stratton's book [20]). However, it is possible to get a grasp on the real physics by utilizing the known theory for a plane laser beam interacting with a dielectric sphere, whose refractive index relative to the surroundings is greater than unity. Light becomes concentrated at the rear region of the sphere as a consequence of the lens effect, resulting in an enhanced electromagnetic energy density, and thus an enhanced surface force density, at the rear.

To illustrate this phenomenon mathematically, let us consider the simplified case of an 
isolated isotropic sphere of radius $a$ and refractive index $n_{2}$ situated in an ambient medium of refractive index $n_{1}$, illuminated by a plane wave incident from above, along the $z$ axis. Taking the wave to be polarized in the $x$ direction, we can represent it in complex notation as

$$
\mathbf{E}^{(i)}=E_{0} \mathbf{e}_{x} e^{i k_{1} z}, \quad \mathbf{B}^{(i)}=\frac{n_{1} E_{0}}{c} \mathbf{e}_{y} e^{i k_{1} z},
$$

where $E_{0}$ is the amplitude and $k_{1}=n_{1} \omega / c$ the incident wave number. The time factor $\exp (-i \omega t)$ has been omitted. We shall need the electric field components on the interior surface of the sphere (superscript $w$ ), at $r=a^{-}$. In standard notation the components can be written 12$]$

$$
\begin{gathered}
E_{r}^{(w)}(a)=\frac{E_{0} \cos \phi}{\left(k_{2} a\right)^{2}} \sum_{l=1}^{\infty} i^{l+1}(2 l+1) a_{l}^{(w)} \psi_{l}\left(k_{2} a\right) P_{l}^{1}, \\
E_{\theta}^{(w)}(a)=-\frac{E_{0} \cos \phi}{k_{2} a} \sum_{l=1}^{\infty} \frac{i^{l}(2 l+1)}{l(l+1)}\left[b_{l}^{(w)} \psi_{l}\left(k_{2} a\right) \frac{P_{l}^{1}}{\sin \theta}-i a_{l}^{(w)} \psi_{l}^{\prime}\left(k_{2} a\right) \frac{d P_{l}^{1}}{d \theta}\right], \\
E_{\phi}^{(w)}(a)=\frac{E_{0} \sin \phi}{k_{2} a} \sum_{l=1}^{\infty} \frac{i^{l}(2 l+1)}{l(l+1)}\left[b_{l}^{(w)} \psi_{l}\left(k_{2} a\right) \frac{d P_{l}^{1}}{d \theta}-i a_{l}^{(w)} \psi_{l}^{\prime}\left(k_{2} a\right) \frac{P_{l}^{1}}{\sin \theta}\right]
\end{gathered}
$$


where $\psi_{l}(x)=x j_{l}(x)$ is one of the Riccati-Bessel functions, $k_{2}=n_{2} \omega / c$, and $a_{l}^{(w)}, b_{l}^{(w)}$ are coefficients to be determined from the boundary conditions at $r=a$. These expressions are complicated and will not be reproduced here. The local surface force density, repulsive when $n_{2}>n_{1}$, becomes

$$
\Pi(\theta, \phi)=\frac{\epsilon_{0}}{2}\left(n_{2}^{2}-n_{1}^{2}\right)\left(E_{t}^{(w)^{2}}+\frac{n_{2}^{2}}{n_{1}^{2}} E_{r}^{(w)^{2}}\right)_{a^{-}},
$$

with $E_{t}^{(w)^{2}}=E_{\theta}^{(w)^{2}}+E_{\phi}^{(w)^{2}}$. By means of the expansions (이 - (10) we can calculate $\Pi(\theta, \phi)$ explicitly. It is convenient to write the force in the form of a series:

$$
\Pi(\theta, \phi)=\frac{\epsilon_{0}}{2}\left(n_{2}^{2}-n_{1}^{2}\right) E_{0}^{2} \sum_{l=0}^{\infty} \sum_{m=-l}^{l} F_{l m} P_{l}^{m}(\cos \theta) e^{i m \phi},
$$

where the constant coefficients $F_{l m}$ can be calculated after inversion of the series. (The $l=0$ term descibes a uniform pressure balance within the sphere.)

As one should expect from the form of the series (12), the surface force density becomes most pronounced for small or moderate values of the polar angle $\theta$ (the backward direction corresponds to $\theta=0$ ). Actually, one can make the local surface force effect visible in practice, if one goes one step further and calculates the hydrodynamic displacement of the spherical surface. This calculation was performed in full in Ref. 12], for the case of a water 
sphere situated in air, and was shown to lead to a considerable displacement at the rear end. The experiment of Zhang and Chang 11] clearly showed the reality of the effect. Beautiful computer-generated illustrations of the concentration of radiation energy at the rear of the sphere can be found in the paper of Barton et al. [21].

Thus, to summarize, we have in the previous subsection, and in the present one, analyzed two different simple models intending to give a rough description of the physics of our experiment. The first, 2D geometrical-optics ray-tracing model, attributed the increased surface force in the tip region (in the case of downward illumination) to the presence of total internal reflection (Fig. 4). The second, cylindrically symmetric wave-optical model, attributed the increased surface force to the lens effect for a sphere, together with the diffraction at the rear end. Neither of these models are complete. However, they share the following important property in common with the real experiment when illumination is taking place from above: Light incident along the symmetry axis in an optically dense medium is refracted or diffracted into an outer optically thin medium through a convex surface. This leads in all cases to an increased surface force, and is the essential physics of the effect.

\section{Dependence on Polarization, and on Angle of Incidence}

Another remark is called for, as regards the effect of different states of polarizations of the incident beam. Usually, when dealing with this kind of situations one tacitly assumes that the incident beam itself, as well as the surface distortion, are azimuthally symmetrical. This is evidently true, if the beam is either nonpolarized or circularly polarized. However, if the beam is linearly polarized, the azimuthal symmetry becomes lost. To what extent does then the lack of symmetry in the case of linear polarization influence the distortion of the 
surface?

To illustrate this point, we show in Fig. 5 the normalized radiation pressure $\frac{\Pi\left(\theta_{1}\right)}{\Pi(0)}$ on a flat surface versus the angle of incidence $\theta_{1}$, when the beam falls from an optically thin $\left(\Phi_{1}\right.$ in our experiment) against an optically thick medium $\left(\Phi_{2}\right)$. The curves, calculated separately for the TM and TE polarizations, follow from Eq. (3) and the Fresnel relations. We see that the sensitivity with respect to shift in polarizations is only moderate, the curves being in fact coincident. For sake of comparison, we show in Inset of Fig 5 the case of a water/air interface, light being incident from air $\left(n_{1}=1\right)$ to water $\left(n_{2}=1.33\right)$. The dependence on polarization is more visible: in the TM case, there is a small enhancement of the pressure near the Brewster angle.

On the other hand, Fig. 6 shows how the optical radiation pressure varies with the angle of incidence, here called now $\theta_{2}$, when the ray is reversed and falls from $\Phi_{2}$ to $\Phi_{1}$. Once again, the dependence upon shift in polarizations is seen to be very small for our experimental conditions, compared to the case of a water/air interface (see Inset). The most notable feature of the curves is the pronounced directional effect: near the angle of total reflection, the pressure becomes enhanced by a factor of $\left(1+n_{1} / n_{2}\right)^{2}$, as compared with the case of normal incidence. This effect is of course the same as that discussed in Secs. III.A and B, but now regarded from a somewhat different angle, as being a direct consequence of Fresnel's equations. It should be noticed that this enhancement of the optical radiation pressure under total reflection condition was exploited by Komissarova et al. in their experiments [3], and also in Ref. 22. Let us note that in our situation $n_{1}$ and $n_{2}$ are very close, due to the vicinity of the critical point. Then, while the total reflection angle is shifted towards larger values, the sensitivity to polarization is even smaller.

As a conclusion, it is justified to consider for our experiment that the force is cylindrically 
symmetric, even for a linearly polarized incident beam. This moderate sensitivity with respect to shift in local polarizations (the local states of polarizations are of course different if we move around in the azimuthal direction on the distorted surface), can be compared with what is observed if the refractive medium is not a dielectric but instead a metal. In the latter case, there is no polarization dependence at all. We refer the reader to the accurate measurement of Jones and Leslie [23] and to the comprehensive treatment given in Jones' monograph 24]. This experiment has been discussed theoretically also in Ref. 16, Appendix. The dependence upon polarization is thus entirely associated with the dielectric property of the medium and is absent if its permittivity goes to infinity.

\section{COMPARISON WITH THE ZHANG-CHANG EXPERIMENT}

First of all, it ought to be emphasized that the use of a laser pulse instead of a continuous beam in this experiment does not make any difference in principle. The surface forces act in the same way in the two cases. It was observed that the deflections of the free surface took place well after the passage of the pulse, but the reason for this is the long hydrodynamic response time. The basic time scale is in fact determined by the transit time of sound, across the dimensions of the medium [16]. Now, for a sphere of radius $a=50 \mu \mathrm{m}$ this is not very long, less than $0.1 \mu \mathrm{s}$. However, the complete displacement of the surface involves a series of multiple internal reflections of sound at the surface, responsible for a longer delay. In the experiment, the surface velocity was found to be appreciable for $10 \mu$ s or more.

This point being specified, as universality is ensured by the criticality of our experimental medium, Eq. (2) should of course be applicable to a flat water/air interface also ( $\sigma \simeq 70 \mathrm{mN} / \mathrm{m}$ at room temperature). Then, despite the presence of curved surfaces, the formation of a liquid fountain at the rear face of the droplet in the experiment of Zhang and 
Chang [11] should be at least roughly explainable in terms of the same instability mechanism. From Eq. (2) we find $P_{S}=29 \mathrm{~kW}$ when $\omega_{0}=100 \mu \mathrm{m}$. Now, it turns out that both the $100 \mathrm{~mJ}$ and the $200 \mathrm{~mJ}$ laser pulses used in the Zhang-Chang experiment overshoot this limit. They correspond respectively to $P=125 \mathrm{~kW}$ and $250 \mathrm{~kW}$ (see Ref. 12).

When taken at face value, these numbers indicate that there is a considerable discrepancy between theory and experiment in the Zhang-Chang case. However, some care ought to be taken here, in view of the fact that Zhang and Chang made use of short laser pulses. Let us for definiteness consider the $100 \mathrm{~mJ}$ pulse, distributed over a cross-sectional disk of radius $100 \mu \mathrm{m}$. It is physically most appropriate to discuss the time-dependent centerline intensity $I(t)$, rather than the total power $P$. The pulse can be modeled as $I(t)=\left(I_{0} t / \tau\right) \exp (-t / \tau)$, where $\tau=0.40 \mu \mathrm{s}$ [12]. When the pulse is plane, $I_{0}$ is constant. With $A=\pi \times 10^{-4} \mathrm{~cm}^{2}$, we obtain $I_{0}=0.80 \mathrm{GW} / \mathrm{cm}^{2}$. The maximum intensity, $I_{\max }=I_{0} / e=0.29 \mathrm{GW} / \mathrm{cm}^{2}$, occurs at $t=\tau$. Taking the effective duration of the pulse to be $3 \tau\left(I(3 \tau)=0.12 \mathrm{GW} / \mathrm{cm}^{2}\right)$, the time-averaged intensity becomes $\bar{I}=0.21 \mathrm{GW} / \mathrm{cm}^{2}$. When this is compared with the intensity of a stationary plane wave with power $P=29 \mathrm{~kW}$ distributed over the same area (this corresponds to $I=0.09 \mathrm{GW} / \mathrm{cm}^{2}$ ), we see that the Zhang-Chang measurements yield a factor of about 2 times the value predicted by Eq. (2). One might here argue, however, that it is physically more appropriate to compare the Zhang-Chang mean power of $0.21 \mathrm{GW} / \mathrm{cm}^{2}$ not with the case of a plane wave, but instead with a Gaussian beam at a point located off the symmetry axis by a distance corresponding to one half of the centerline intensity maximum. A Gaussian beam can be modeled, in the waist plane, as $I(r, t)=I(r) T(t)$, where 


$$
I(r)=\frac{2 P}{\pi \omega_{0}^{2}} \exp \left(-2 r^{2} / \omega_{0}^{2}\right), \quad T(t)=\frac{t}{\tau} \exp (-t / \tau)
$$

(The half-maximum intensity is seen to correspond to $r=0.59 \omega_{0}$.) According to this the Gaussian centerline value should be doubled, from 0.09 to about $0.20 \mathrm{GW} / \mathrm{cm}^{2}$, and the agreement with the Zhang-Chang experiment becomes quite good.

The above estimate illustrates to what level we can expect agreement between theory and experiment. There are at least three reasons why we cannot expect large accuracy. First, there is the roughness of the calculation. Secondly, there is the fact that the original undisturbed surface in the Zhang-Chang experiment was already curved. Total internal reflections could thus occur in that case. In the present case, without any distortion caused by the beam itself, there will not be any total internal reflections. Finally, it is to be recalled that Eq. (2) is dealing with the limiting case of onset of total reflection only. It is conceivable that some more power is required before the large displacement of the surface develops in full. This is confirmed by dynamical investigations of the temporal development of the instability 25]. The last-mentioned point becomes applicable here because Zhang and Chang reported the instability to occur in the region between 100 and $200 \mathrm{~mJ}$.

\section{FINAL REMARKS AND CONCLUSIONS}

\section{A. On the Influence from Electrostriction}

When considering the electromagnetic forces above, we neglected the effect from electrostriction. To see the legitimacy of this neglect more closely, let us briefly consider 
the expression for the full electromagnetic force density in a nonmagnetic fluid (except from the fluctuating Abraham term)

$$
\mathbf{f}=-\frac{1}{2} E^{2} \nabla \varepsilon+\frac{1}{2} \nabla\left(E^{2} \rho \frac{d \varepsilon}{d \rho}\right)
$$

where $\rho$ is the mass density (cf., for instance, Refs. 12,16,20). Here the second term represents electrostriction. Calculating this term by means of the Clausius-Mossotti equation, we get

$$
\mathbf{f}=-\frac{\varepsilon_{0}}{2} E^{2} \nabla \kappa+\frac{\varepsilon_{0}}{6} \nabla\left[E^{2}(\kappa-1)(\kappa+2)\right]
$$

$\kappa=\varepsilon / \varepsilon_{0}$ being the relative permittivity. The electrostriction pressure is always compressive. The reason why it usually is left out in practical calculations, is that it does not contribute to the total force on a test body. When this kind of force compresses the body, there is quickly established a counterbalancing elastic pressure in the interior, so that the net influence on the body vanishes. The relevant time scale for the establishment of this counterbalancing pressure is the time that sound needs to traverse the illuminated region. For instance, if we estimate the transverse scale to be $10 \mu \mathrm{m}$ we see, when taking the velocity of sound to be $1500 \mathrm{~m} / \mathrm{s}$, that the relevant time scale becomes about $7 \mathrm{~ns}$. Figure 9 in Ref. 16 shows, as a result of a detailed calculation, how the elastic compensation 
in water becomes established in a very few nanoseconds. The thesis of Poon [26] may also be consulted for a very detailed analysis of these effects. Our conclusion is thus that the electrostriction effect can safely be left out in the present case, as we are dealing with stationary deformations.

\section{B. Conclusion}

The main purpose of the present work has been to experimentally show that LaserInduced-Surface-Deformations become asymmetric at high field strength. This asymmetry is characterized for the first time by using near-critical liquid-liquid interfaces to strongly enhance optical radiation pressure effects. The mechanisms at the origin of this asymmetry are presented and illustrated with very simple arguments. In particular, we demonstrate that the dependence of optical radiation pressure versus the angle of incidence (Eq. (3)) should be taken into account. This point, which is generally neglected in the case of classical liquid interfaces, could be a first step to explain the surprising tether shapes observed. Some

preliminary calculations seem to confirm it [27], even if the numerical scheme used need to be refined.

In the case leading to interface instability, we also compare our experimental results with those obtained by Zhang and Chang for water droplets under laser pulses excitation. This comparison is theoretically justified because near-criticality leads to an universal description of radiation pressure effects. The predicted power onset turn out to be of the same order than that observed with pulsed excitation.

Finally, since the laser light propagates under total reflection condition inside the induced filament, this one provides, to the best of our knowledge, the first example of non-permanent self-written liquid waveguide. 


\section{Acknowledgments}

We are grateful to Max Winckert and Jöel Plantard for technical assistance. This work was partly supported by the CNRS and the Conseil Régional d'Aquitaine.

[1] A.Ashkin and J.M. Dziedzic, "Radiation pressure on a free liquid surface", Phys. Rev. Lett. 30, 139 (1973).

[2] A. V. Kats and V. M. Kontorovich, "Lens effect due to the pressure of light on the surface of a transparent liquid", Sov. Phys.-JETP 41, 346 (1975).

[3] I. I. Komissarova, G. V. Ostrovskaya and E. N. Shedova, "Light pressure-induced deformations of a free liquid surface", Optics Commun. 66, 15 (1987).

[4] J. M. Hartings, X. Pu, J. L. Cheung and R.K. Chang, "Laser-induced distorsion for increased input coupling of light to droplet-cavity modes", J. Opt. Soc. Am. B 14, 2842 (1997).

[5] J. Guck, R. Ananthakrishnan, T. J. Moon, C. C. Cunningham and J. Käs, "Optical deformability of soft biological dielectrics" Phys. Rev. Lett. 84, 5451 (2000).

[6] J. Guck, R. Ananthakrishnan, H. Mahmood, T. J. Moon, C. C. Cunningham and J. Käs, "The optical stretcher: a novel tool to micromanipulate cells", Biophys. J. 84, 767 (2001).

[7] C. H. Lee, C. L. Guo and J. Wang, "Optical measurement of the viscoelastic and biochemical responses of living cells to mechanical perturbation", Opt. Lett. 23, 307 (1998).

[8] C. H. Lee, W. C. Lin and J. Wang, "All-optical measurements of the bending rigidity of lipid-vesicle membranes across structural phase transitions", Phys. Rev. E 64, 020901 (2001).

[9] K. Sakai, D. Mizuno and K. Takagi, "Measurement of liquid surface properties by laser-induced surface deformation spectroscopy", Phys. Rev. E 63, 046302 (2001). 
[10] S. Mitani and K. Sakai, "Measurement of ultralow interfacial tension with a laser interface manipulation technique", Phys. Rev. E 66, 031604 (2002).

[11] J.Z. Zhang and R.K. Chang, "Shape distorsion of a single water droplet by laser-induced electrostriction", Opt. Lett. 13, 916 (1988).

[12] I. Brevik and R. Kluge, " Oscillations of a water droplet illuminated by a linearly polarized laser pulse", J. Opt. Soc. Am. B 16, 976 (1999).

[13] R. Loudon, The Quantum Theory of Light (Clarendon Press, Oxford, 1986), p. 348.

[14] A. Casner and J. P. Delville, "Giant deformations of a liquid-liquid interface induced by the optical radiation pressure", Phys. Rev. Lett. 87, 054503 (2001).

[15] H. M. Lai, P. T. Leung, K. L. Poon and K. Young, "Electrostrictive distortion of a micrometersized droplet by a laser pulse", J. Opt. Soc. Am. B 6, 2430 (1989).

[16] I. Brevik, "Experiments in phenomenological electrodynamics and the electromagnetic energymomentum tensor", Physics Reports 52, 133 (1979).

[17] A.Casner and J. P. Delville, "Adaptative lensing driven by the radiation pressure of a continuous-wave laser wave upon a near-critical liquid-liquid interface", Opt. Lett. 26, 1418 (2001).

[18] A.Casner and J. P. Delville, "Laser-induced hydrodynamic instability of fluid interfaces", Phys. Rev. Lett. 90, 144503 (2003).

[19] G. N. Borzdov, "An intrinsic tensor technique in Minkowski space with applications to boundary value problems", J. Math. Phys. 34, 3162 (1993).

[20] J. A. Stratton, Electromagnetic Theory (McGraw-Hill, New York, 1941).

[21] J. P. Barton, D. R. Alexander, and S. A. Schaub, "Internal and near-surface electromagnetic fields for a spherical particle irradiated by a focused laser beam", J. Appl. Phys. 64, 1632 
(1988).

[22] O. N. Ivanova, S. P. Chernov and V. A. Shepelev, "Experimental investigation of the interaction of high-power laser radiation with the free surface of a liquid under total internal reflection conditions", Sov. J. Quant. Electron. 4, 1161 (1975).

[23] R. V. Jones and B. Leslie, "The measurement of optical radiation pressure in dispersive media", Proc. Roy. Soc. London A 360, 347 (1978).

[24] R. V. Jones, Instruments and Experiences, (John Wiley, New York, 1988).

[25] A.Casner and J. P. Delville, personal communication.

[26] K. L. Poon, "Laser pulse induced electrostrictive distortion of liquid micro-droplet", M. Phil. thesis (Chinese University of Hong Kong, Hong Kong, 1990).

[27] A. Casner, "Déformations, manipulations et instabilités d'interfaces liquides induites par la pression de radiation d'une onde laser", PhD thesis (Université Bordeaux I, Bordeaux, France, 2001). http://tel.ccsd.cnrs.fr/documents/archives0/00/00/16/37/index_fr.html 


\begin{tabular}{|c|c|c|c|c|c|c|c|}
\hline$n_{0}$ & $\Delta n$ & $\rho_{0}\left(\mathrm{~kg} \cdot \mathrm{m}^{-3}\right)$ & $\Delta \rho\left(\mathrm{kg} \cdot \mathrm{m}^{-3}\right)$ & $\sigma\left(\mathrm{J} . \mathrm{m}^{-2}\right)$ & $\alpha_{t h}\left(\mathrm{~cm}^{-1}\right)$ & $\Lambda_{t h}\left(W \cdot \mathrm{cm}^{-1} \cdot \mathrm{K}^{-1}\right)$ & $D_{t h}\left(\mathrm{~cm}^{2} \cdot \mathrm{s}^{-1}\right)$ \\
\hline \hline 1.464 & $-7.610^{-3}$ & 872 & 63.3 & $310^{-7}$ & $310^{-4}$ & $1.2810^{-3}$ & $8.810^{-4}$ \\
\hline
\end{tabular}

TABLE I: Experimental parameters of our medium. $n_{0}$ and $\rho_{0}$ : mean refractive index and density for $T<T_{C} . \Delta n, \Delta \rho, \sigma:$ refractive index contrast, density index contrast and surface tension evaluated at $T-T_{C}=3 K . \alpha_{t h}, \Lambda_{t h}$ and $D_{t h}$ : optical absorption, thermal conductivity and thermal diffusivity.

\section{Table}




\section{List of Figure Captions}

Fig. 1

Experimental set-up. BS: beam splitter, L: lens, M1,M2,M3: dielectric mirrors, $\frac{\lambda}{2}: \frac{\lambda}{2}$ plate, C: thermoregulated spectroscopic cell.

Fig. 2

Interface deformations induced at $\left(T-T_{C}\right)=3 K$ by a laser beam of waist $\omega_{0}=5.3 \mu m$.(a) Laser propagating upwards from $\Phi_{1}$ to $\Phi_{2}$ as indicated by the white arrow. $P$ increases from top to bottom and is successively equal to 210, 270, 300, 410, 530, 590 and 830 mW. (b) Downward direction of propagation. $P=190,250,280,310,340,370,400$ and $400 \mathrm{~mW}$. The two last pictures are snapshots showing the destabilization of the interface at $P_{S}$ leading to the formation of a stationary jet similar to that illustrated in (c) for $\left(T-T_{C}\right)=6 K, \omega_{0}=3.5 \mu \mathrm{m}$ and $P=700 \mathrm{~mW} . P_{S}=490 \mathrm{~mW}$ in this last case. The total height of picture (c) is $1 \mathrm{~mm}$.

Fig. 3

Evolution of the centerline height of the deformation $h_{0}=h(r=0)$ versus $P$ corresponding to the pictures of Fig. 2a) ( $)$ and Fig 2b) $(\triangle)$. Broken line indicates the onset $P_{S}$ above which the interface becomes unstable when the laser is propagating downward.

Fig.4

Sketch of symmetric ray track when the beam is incident from above. Angle of incidence $\theta_{2}=\beta=45^{0} ; n_{2} / n_{1}=\sqrt{2}$. 
Fig.5

Normalized radiation pressure on flat surface versus angle of incidence $\theta_{1}$ for the TM and TE polarizations, when $T-T_{C}=3 K$ as in experiment. The light right is incident from $\Phi_{1}$ to $\Phi_{2}$. Inset: same curves drawn for an air/water interface, ray incident from air $\left(n_{1}=1\right)$ to water $\left(n_{2}=1.33\right)$.

Fig. 6

Same as Fig. 5, but with direction of ray reversed, from $\Phi_{2}$ to $\Phi_{1}$, or for the Inset from water to air. Angle of incidence is now denoted by $\theta_{2}$. 


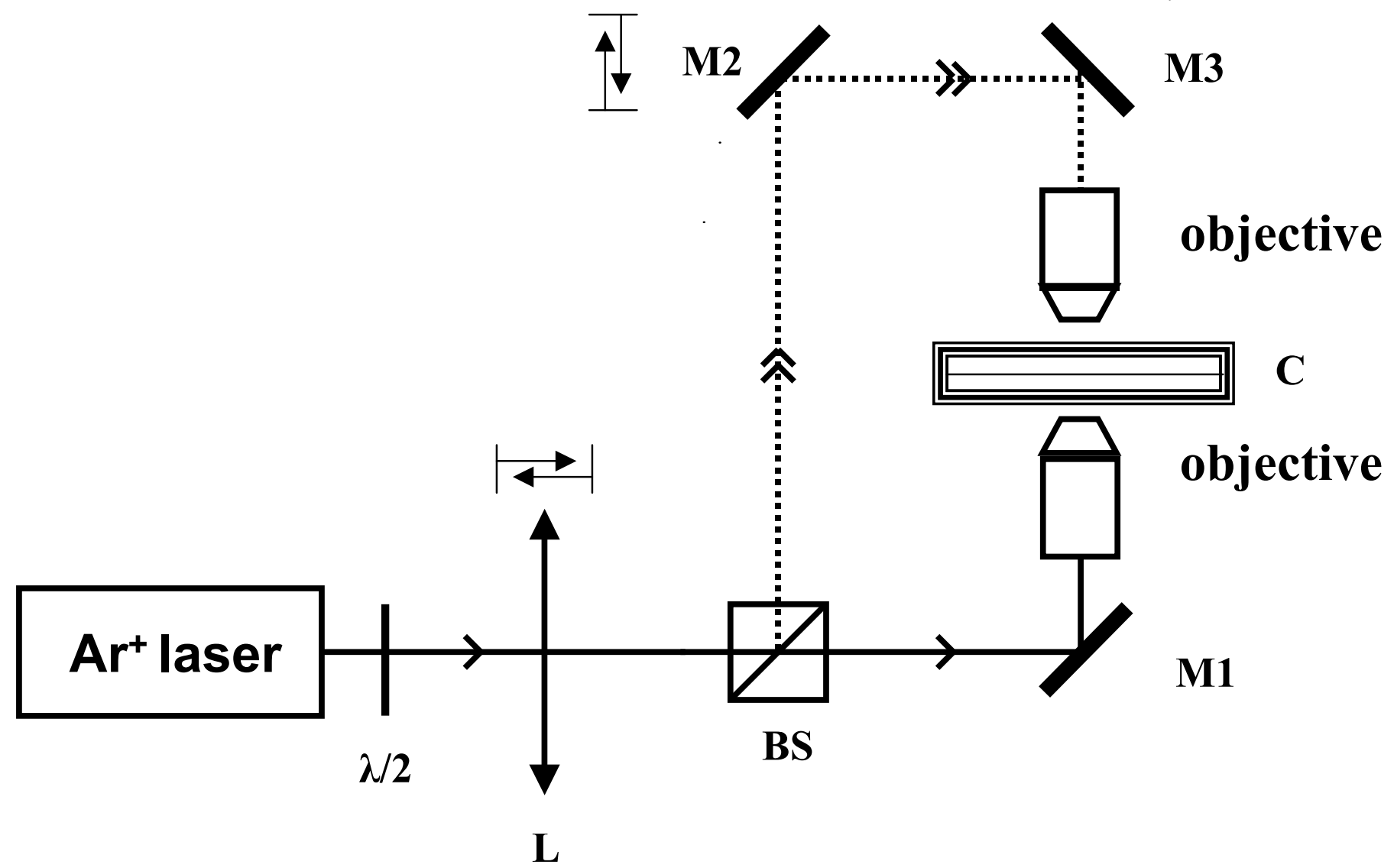

Fig. 1 


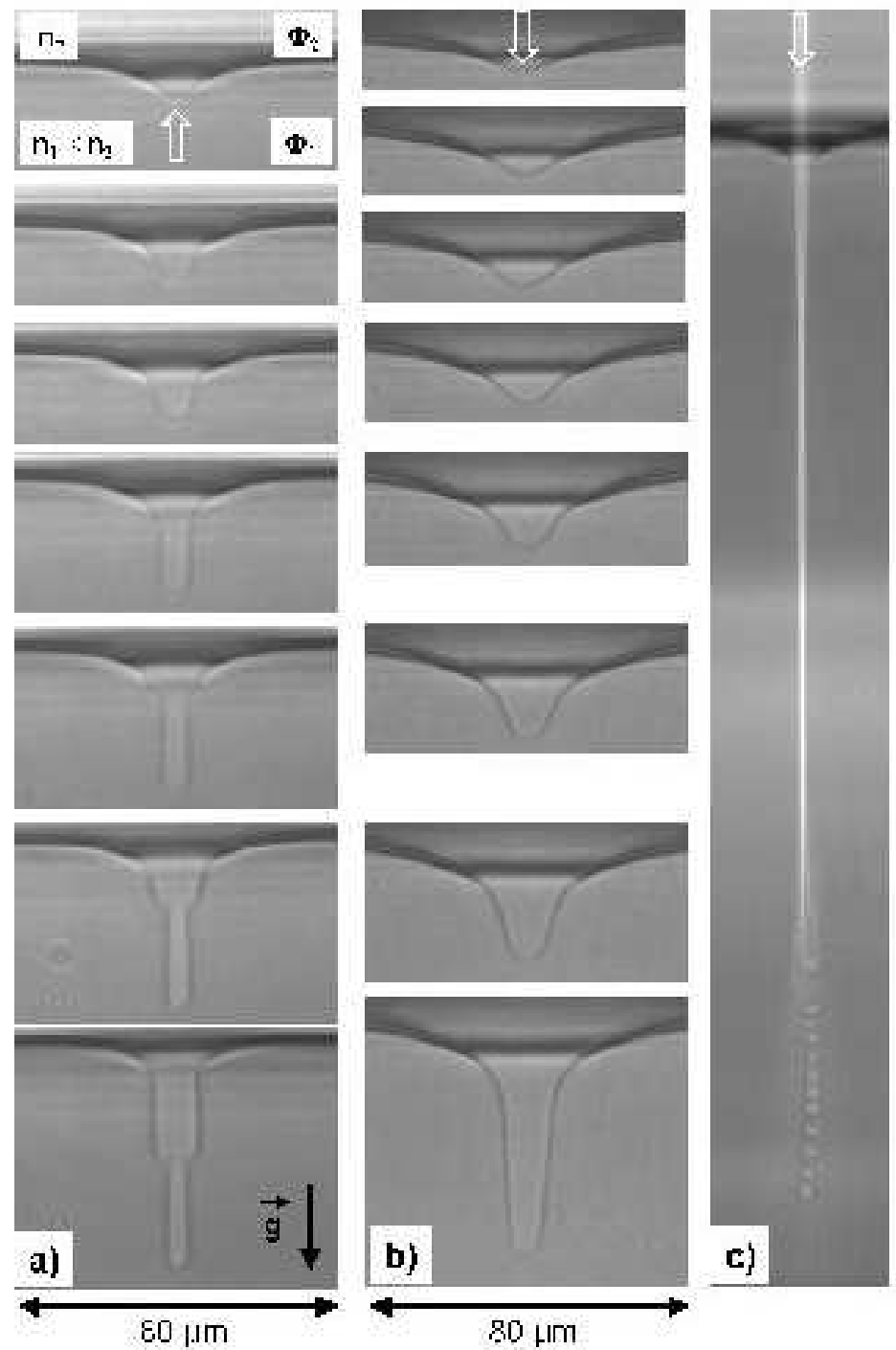

Fig. 2 


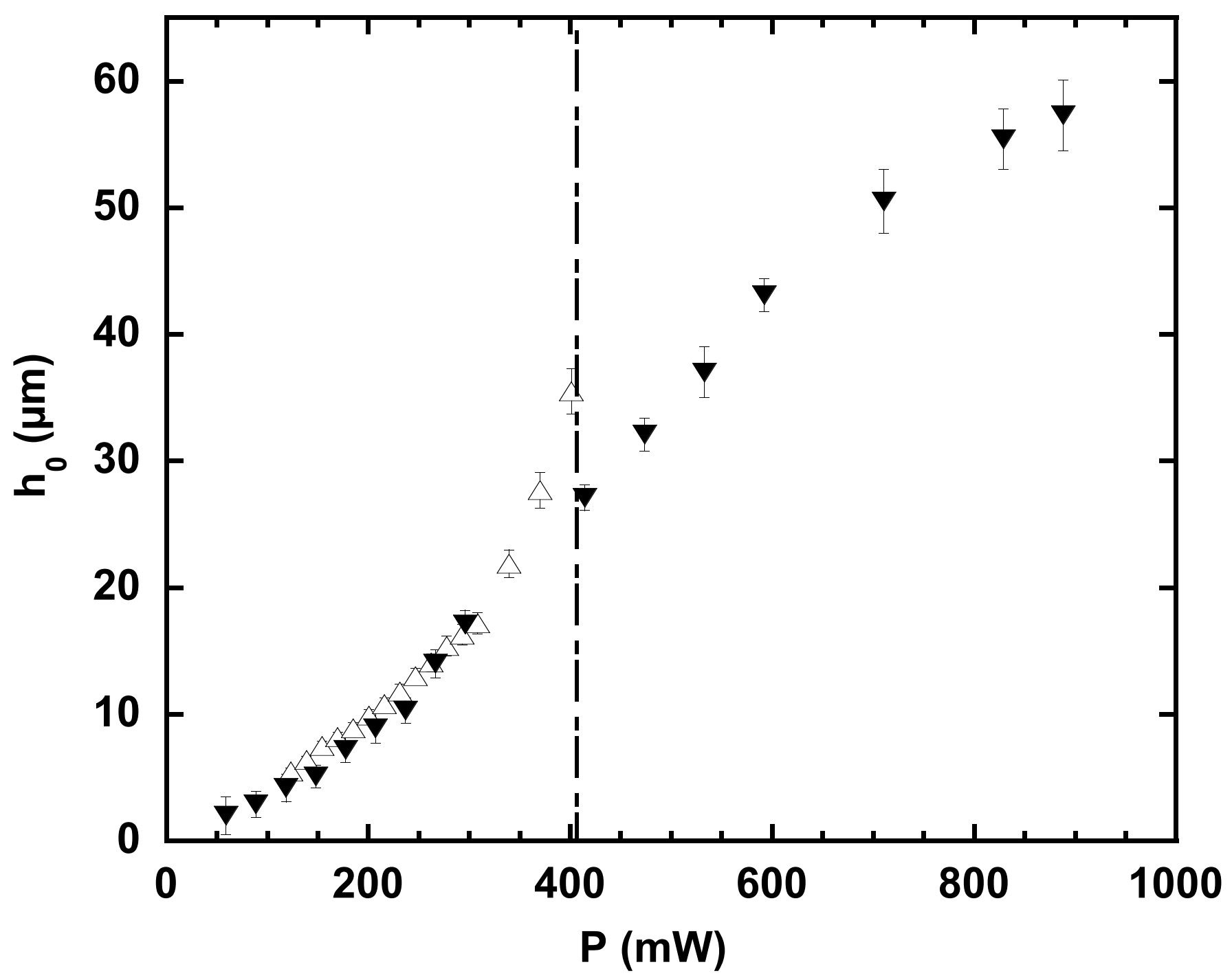

Fig. 3 


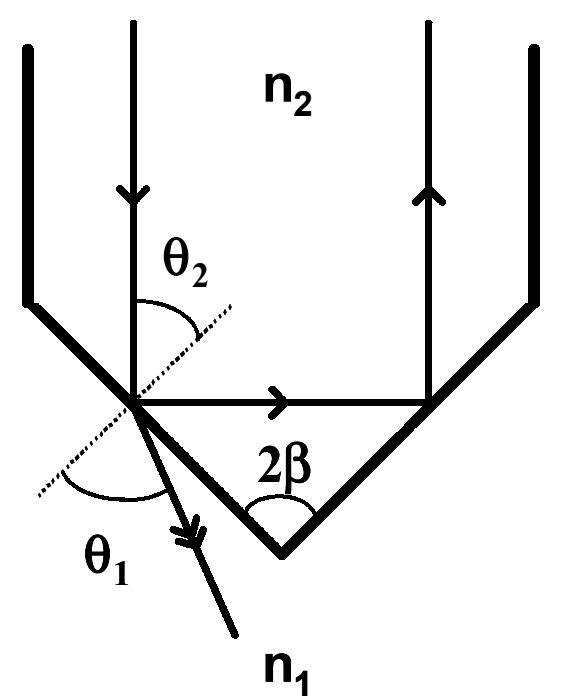

Fig. 4 


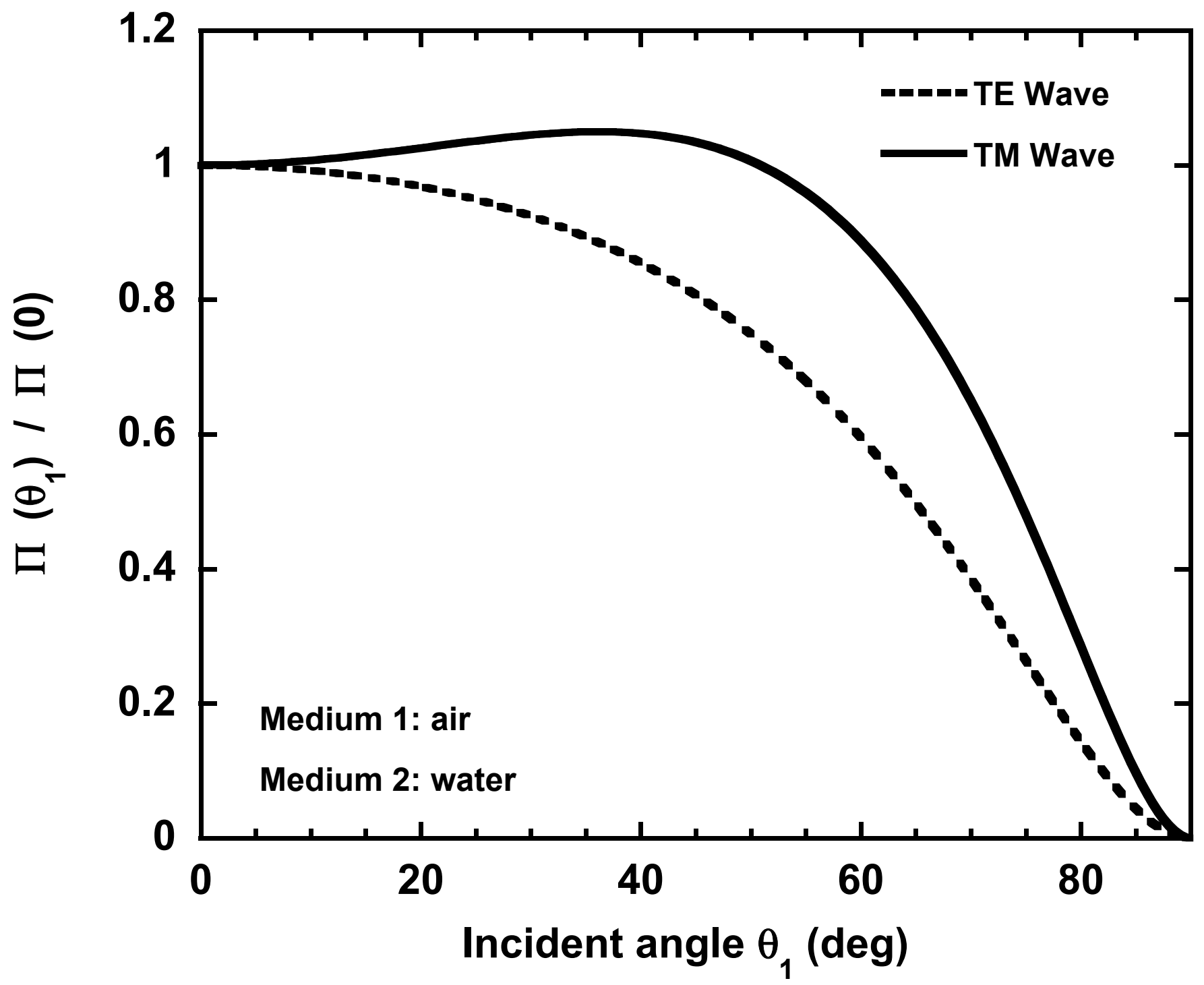

Fig. 5 


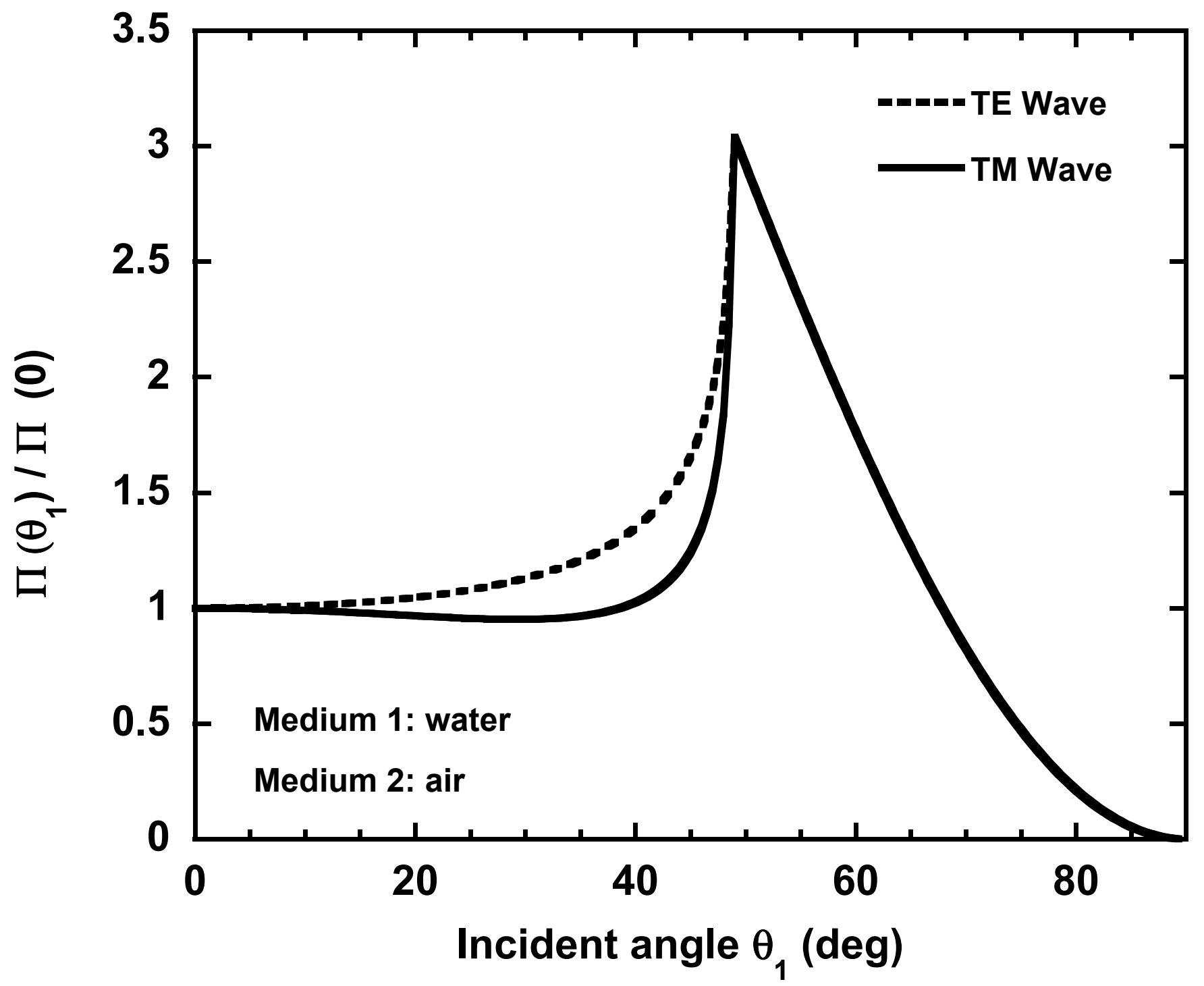

Fig. 6 